\title{
Flow over Hills: A Large-Eddy Simulation of the Bolund Case
}

\author{
Marc Diebold • Chad Higgins • \\ Jiannong Fang • Andreas Bechmann • \\ Marc B. Parlange
}

Received: 14 June 2012 / Accepted: 5 February 2013

(C) Springer Science+Business Media Dordrecht 2013

\begin{abstract}
Simulation of local atmospheric flows around complex topography is important for several applications in wind energy (short-term wind forecasting and turbine siting and control), local weather prediction in mountainous regions and avalanche risk assessment. However, atmospheric simulation around steep mountain topography remains challenging, and a number of different approaches are used to represent such topography in numerical models. The immersed boundary method (IBM) is particularly well-suited for efficient and numerically stable simulation of flow around steep terrain. It uses a homogenous grid and permits a fast meshing of the topography. Here, we use the IBM in conjunction with a largeeddy simulation (LES) and test it against two unique datasets. In the first comparison, the LES is used to reproduce experimental results from a wind-tunnel study of a smooth threedimensional hill. In the second comparison, we simulate the wind field around the Bolund Hill, Denmark, and make direct comparisons with field measurements. Both cases show good agreement between the simulation results and the experimental data, with the largest disagreement observed near the surface. The source of error is investigated by performing additional simulations with a variety of spatial resolutions and surface roughness properties.
\end{abstract}

M. Diebold ( $\varangle) \cdot$ J. Fang · M. B. Parlange

School of Architecture, Civil and Environmental Engineering,

Ecole Polytechnique Fédérale de Lausanne, 1015 Lausanne, Switzerland

e-mail: marc.diebold@epfl.ch

J. Fang

e-mail: jiannong.fang@epfl.ch

M. B. Parlange

e-mail: marc.parlange@epfl.ch

C. Higgins

Department of Biological and Ecological Engineering,

Oregon State University, Corvallis, OR 97331, USA

e-mail: Chad.Higgins@oregonstate.edu

A. Bechmann

Department of Wind Energy, Technical University of Denmark, 4000 Roskilde, Denmark e-mail: andh@dtu.dk 
Keywords Bolund Hill - Complex terrain - Computational fluid dynamics - Immersed boundary method · Large-eddy simulation · Navier-Stokes equation · Topography · Validation of computational fluid dynamics models

\section{Introduction}

Accurate wind field modelling in complex topography has many relevant applications. For example, wind fields are essential in evaluating wind power potential, for formulating real time responses to accidental releases of hazardous materials in urban areas, and for assessing the redistribution of the alpine snowpack and resulting avalanche risk. In all of these cases, the wind field must be evaluated around topographic elements such as buildings or mountains. This creates unique numerical challenges, i.e. prescribing the proper boundary conditions while also solving a high Reynolds number turbulent flow. Steep slopes challenge numerical methods (Tseng and Ferziger 2003). The immersed boundary method (IBM) (Peskin 1972, 2002; Mittal and Iaccarino 2005) is a numerical technique that can be used to incorporate topography into conventional simulation approaches. It is well suited for environmental applications in which the terrain is steep. In regard to turbulence, direct numerical simulation (DNS), which resolves the full turbulence spectra of atmospheric flows, is not practical with currently available computational power (Voller and Porte-Agel 2002). Large-eddy simulation (LES) (Deardorff 1970; Bou-Zeid et al. 2004) is a numerical technique in which largescale turbulent motion is resolved and small-scale motion is numerically parametrized. Here, we couple the IBM with LES to simulate the turbulent flow around topographic elements.

The following section describes LES and IBM techniques along with details concerning the numerical model. We then compare simulation results with measurements. In Sect. 4, a wind-tunnel experiment of flow around a steep three-dimensional hill is described, and the experimental results are compared directly to LES. Section 5 describes a 2009 field campaign on the Bolund Hill in Denmark; these field measurements are likewise compared with LES results. In both cases we find favourable agreement between measurements and simulation results. Finally, the boundary conditions and the resolution of the simulation are modified to investigate the sources of error and identify potential improvements.

\section{Model Description}

\subsection{Large-Eddy Simulation}

Currently available computational power is not sufficient to resolve all of the relevant scales of turbulent motion $(\mathrm{O}(1 \mathrm{~mm})-\mathrm{O}(10 \mathrm{~km}))$ in the atmosphere. Nonetheless, a substantial portion of the relevant scales of motion can be resolved. In the atmosphere, the largest scales of motion are the most energetic and are responsible for the majority of turbulent transport. The smallest scales of motion are more isotropic and thus amenable to parametrization. This dichotomy provides the conceptual foundation of LES in which turbulent motions are separated into resolved scales that are computed on a grid of a given resolution, and smaller, so-called subgrid scales (SGS) that can be parametrized (Lilly 1967). Applying a filtering operation to the Navier-Stokes equations results in the LES equations:

$$
\frac{\partial \tilde{u}_{i}}{\partial t}+\tilde{u}_{j} \frac{\partial \tilde{u}_{i}}{\partial x_{j}}=-\frac{1}{\rho} \frac{\partial \tilde{p}}{\partial x_{i}}-\frac{1}{\rho} \frac{\partial \tau_{i j}}{\partial x_{j}},
$$




$$
\frac{\partial \tilde{u}_{i}}{\partial x_{i}}=0,
$$

where $\tau_{i j}=\widetilde{u_{i} u_{j}}-\tilde{u_{i}} \tilde{u_{j}}$ is the SGS stress tensor that must be parametrized. In these equations, the tilde represents the filtering operation, $x_{i}$ represent the three spatial axes, $u_{i}$ represent the three velocity components, $\rho$ is the air density and $p$ is pressure. Many models have been developed to deal with the SGS terms. The first, and most popular, the so-called Smagorinsky model (Smagorinsky 1963), uses a mixing length approach:

$$
\tau_{i j}^{\text {Smag }}=-2 C_{\mathrm{s}}^{2} \Delta^{2}|\tilde{S}| \tilde{S_{i j}},
$$

in which $C_{\mathrm{s}}$ is the Smagorinsky constant, $\Delta$ is the filter length and $S_{i j}$ is the strain rate tensor defined by $S_{i j}=\frac{1}{2}\left(\frac{\mathrm{d} u_{i}}{\mathrm{~d} x_{j}}+\frac{\mathrm{d} u_{j}}{\mathrm{~d} x_{i}}\right)$. For this model formulation, the central issue is the proper choice of the Smagorinsky constant $C_{\mathrm{s}}$, which can be computed dynamically (Germano et al. 1991; Porte-Agel et al. 2000; Meneveau and Katz 2000) using a Lagrangian scale-dependent implementation (Bou-Zeid et al. 2005).

If the simulation includes topography, a more appropriate SGS model might be the Lagrangian model, which allows for a more natural averaging defined by the fluid flow, where the Eulerian model simply averages over a plane. These different approaches have been compared in the literature (Nieuwstadt et al. 1991; Andren et al. 1994) and simulation results have been verified against field measurements over topographic elements such as the Askervein Hill (Taylor and Teunissen 1987) and the Bolund Hill (Bechmann et al. 2009; Berg et al. 2011).

The LES model from Ecole Polytechnique Fédérale de Lausanne (EPFL-LES) was developed from the original model described by Albertson and Parlange (1999). In this work, the simulations were performed using the scale-dependent Lagrangian dynamic subgrid model developed by Bou-Zeid et al. (2005). To compute the Smagorinsky coefficient, $C_{\mathrm{s}}$, the scaledependent model uses two filters at sizes twice and four times the LES grid size $\Delta$. Therefore, $C_{\mathrm{s}, 2 \Delta}$ and $C_{\mathrm{s}, 4 \Delta}$ are computed for the two grid filters and a scaling relationship between the two scales is assumed by $\beta=C_{\mathrm{s}, 4 \Delta}^{2} / C_{\mathrm{s}, 2 \Delta}^{2}$. This result is then used to compute $C_{\mathrm{s}, \Delta}$. The local values of $C_{\mathrm{s}}$ are then averaged in space. If the flow is homogeneous and encounters no obstacles or surface roughness changes, a planar averaging can be performed. This is not the case in the current study where topography is included. Thus, the averaging is done following a Lagrangian scheme (following the path lines of the fluid). The EPFL-LES uses the second-order Adams-Bashforth scheme, in neutral conditions and without buoyancy or Coriolis effects. Spectral methods are used in the horizontal directions coupled with fast Fourier transform to solve the equations. Other methods, such as the iterative Kyrlov solver (Saad 1981) exist but are not considered here. The flow is forced with a static pressure gradient and it is assumed that the Reynolds number of the flow is sufficiently high that the molecular viscosity may be neglected.

\subsection{Immersed Boundary Method}

The most common numerical technique used to implement topography in atmospheric simulations is the terrain-following coordinate system (Gal-Chen and Somerville 1975). However, other methods have been developed to implement an obstacle in a simulation, including the bluff-body technique (Tseng et al. 2006) and the immersed boundary method (IBM), which was first proposed by Peskin (1972) and is still widely used in biological fluid mechanics (Peskin 2002; Mittal and Iaccarino 2005). Using IBM with LES is discussed by Balaras (2004); in this case, SGS elements were modelled dynamically (Germano et al. 1991). The 
efficiency and the low computational cost of IBM compared to terrain-following techniques (body-fit meshes) is discussed in Cristallo and Verzicco (2006). A full description of the implementation of IBM in the EPFL-LES model is given in Chester et al. (2007). Flow is computed on a homogenous grid, and the topography is described separately with a level set function. To define the level set, every point of the computational grid is associated with the shortest distance to the topographic surface by a signed distance function, $\phi(x)$. The value is positive if the point is in the free atmosphere and negative if it is within a topographic element. During the simulation, body forces are applied to all points within the topography (i.e. $\phi(x) \leq 0)$ to prevent flow. A layer above the topographic surface with thickness $\delta=1.1 \Delta$, where $\Delta$ is the grid spacing, is defined. For each point in this layer, a new coordinate system is then created, using the flow direction, the wall normal and their vectorial product. The velocity at a distance $\delta$ from the wall is defined by $\mathbf{v}$. In this new coordinate system, the wall stress is found using a logarithmic wind law:

$$
\tau_{w}=-\rho\left[\frac{\kappa\left|\mathbf{v}_{t}\right|}{\ln \left(1+\delta / z_{0}\right)}\right]^{2},
$$

where $z_{0}$ is the aerodynamic roughness length, $\kappa=0.4$ is the von Karman constant, $\delta$ is the distance to the surface and $\mathbf{v}_{t}$ is the tangential component of the velocity $\mathbf{v}$. A second layer is defined under the surface, in the area $-\delta \leq \phi(x)<0$, in which the stress is extrapolated to ensure a smooth boundary transition. For the remaining points with $\phi(x)<\delta$, the stress profile is smoothed using five successive overrelaxation iterations (Ferziger and Péric 1999) that blend with the stresses at the points close to the immersed boundary and thus yield to a stress field smooth enough to be compatible with the spectral differentiation. Chester et al. (2007) stress that this results in a smearing of the variables over length scales comparable to the grid scale, leading to a loss of accuracy near the surface.

Because the EPFL-LES model is designed with periodic boundary conditions and we require flow fields for a single obstacle, an alternative strategy to enforce the inflow boundary condition is needed. Therefore, the simulation is performed in two steps. First, a precursor simulation is run to acquire a well-defined, time-varying, turbulent inflow and these velocity components are then stored. During this simulation, no topography is present and the flow is forced through the domain along the $x$-axis using a constant mean pressure gradient: $f_{p}=-\mathrm{d} \bar{p} / \mathrm{d} x>0$ per unit volume, where $\bar{p}$ is the mean pressure. This precursor simulation provides the inflow boundary conditions as well as the initial conditions for the main simulation with topography. The last eighth of the domain is used as a buffer region; here, we force the velocity back to the stored velocity components from the precursor simulation: $\tilde{u}(0, y, z)=\tilde{u}_{p}(y, z)$, where $x=0$ is the inlet coordinate of the domain and $\tilde{u}_{p}(y, z)$ denotes the velocity from the precursor simulation. In the buffer area $(7 L / 8 \leq x \leq L, L$ being the size of the domain), the velocity is imposed according to $\tilde{u}(x, y, z)=\tilde{u}(7 L / 8, y, z)+w(x)\left(\tilde{u}_{p}(y, z)-\tilde{u}(7 L / 8, y, z)\right)$, where $w(x)=\frac{1}{2}(1-\cos (\pi(x-7 L / 8) /(L / 8)))$. This allows us to simulate non-periodic flow in the $x$-direction using pseudospectral numerics.

A typical simulation with 256 nodes in each horizontal direction and 128 nodes in the vertical takes about 12 days using 64 processors. The domain is divided into horizontal slices and each processor is assigned one or several of these slices. The bulk of the computing time is devoted to creating a converged inflow dataset. The simulation with topography is completed in $24 \mathrm{~h}$, computing 40,000 timesteps. 


\section{Wind-Tunnel Comparison}

\subsection{Experimental Set-up}

A first test of the EPFL-LES was performed by comparing simulation results with data from an idealized wind-tunnel experiment conducted by Ishihara et al. (1999). A smooth three-dimensional (3D) hill was placed in a wind-tunnel, and wind profiles were measured at seven locations along the centre hill axis. Horizontal profiling was also performed at several locations above the hill and in its wake. The wind speed outside the boundary layer was maintained at $5.8 \mathrm{~m} \mathrm{~s}^{-1}$ and monitored throughout the experiment. This dataset is one of the few that investigates flow around 3D hills in a wind tunnel. Several other wind tunnel experiments focus on two-dimensional hills (Loureiro et al. 2009). In addition, many previous comparisons between LES and wind-tunnel data focus exclusively on vertical profiles (Brown et al. 2001; Allen and Brown 2002; Tamura et al. 2007). The main advantage of the Ishihara dataset is that comparisons can also be made along the horizontal plane. In this study, the hill is described by the equation:

$$
z(x, y)=H \cos ^{2}\left(\frac{\pi \sqrt{x^{2}+y^{2}}}{2 l}\right),
$$

with the hill height $H=40 \mathrm{~mm}$ and $l=100 \mathrm{~mm}$. This topography and the $5.8 \mathrm{~m} \mathrm{~s}^{-1}$ windspeed result in a Reynolds number $(\operatorname{Re}=U L / v$, where $v$ is the kinematic viscosity of the fluid, here air) around 14,500, which is in the lower part of the turbulent range. The wind field was then measured in seven different locations along the flow axis: five points on the hill and two points downstream from the hill. A sampling time of $60 \mathrm{~s}$ was used for mean velocities and turbulence statistics. Horizontal transects of the flow in the wake region are also presented.

\subsection{Simulation Setup}

The LES is performed in a $0.64 \mathrm{~m} \times 0.64 \mathrm{~m}$ horizontal and $0.32 \mathrm{~m}$ vertical domain, in which $x$ is the streamwise direction and $z$ the vertical one. The domain was discretized into $256 \times$ $256 \times 128$ points, resulting in a resolution of $\mathrm{d} x=\mathrm{d} y=\mathrm{d} z=2.5 \mathrm{~mm}$. The roughness length was set to $0.025 \mathrm{~mm}$ to match the conditions in the wind-tunnel experiment. The simulation had a stress-free upper boundary condition, which is not representative of the conditions found in a wind tunnel, but the distance from the top of the hill to the top of the domain is much larger than the size of the hill, so the upper boundary condition does not have a significant impact on the flow in the vicinity of the topography. The simulation's domain height is eight hill heights, and we observe the same phenomenon as Yue et al. (2007). About three hill heights above the topography, the flow returns to an undisturbed logarithmic profile. The LES results were averaged in time over $2 \mathrm{~s}$ and compared with the experimental values, representing 80,000 iterations.

\subsection{Comparison}

The results of the comparison between the measured wind-tunnel flow velocities and the computed LES flow velocities are presented in Fig. 1a. Upwind of the peak, measurements and simulation results agree well, but on the lee side of the hill, differences between the LES and the measurements are apparent. The comparison becomes more favourable at greater 

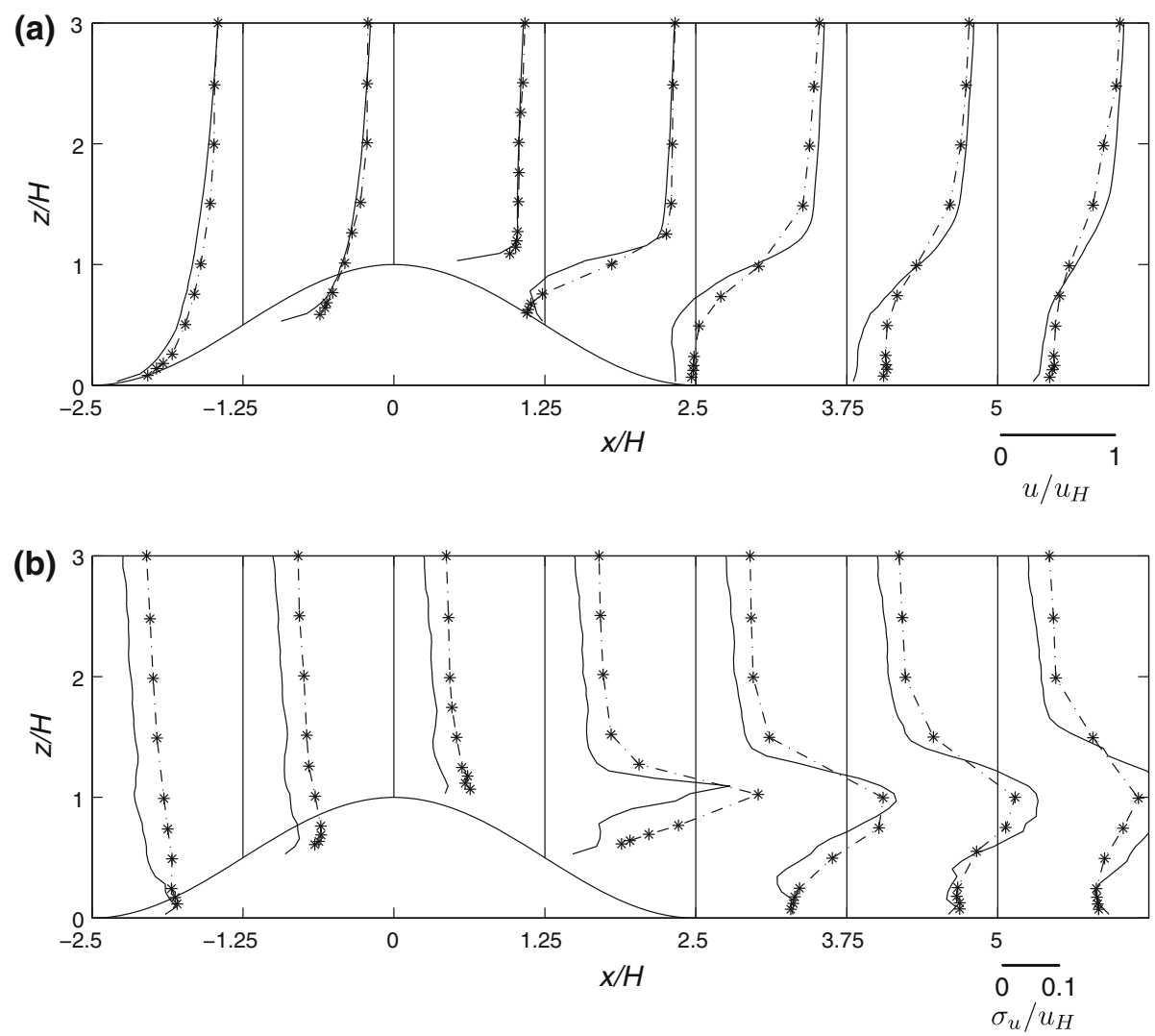

Fig. 1 Stars are the wind-tunnel measurements, the solid line represents the LES results. Data are given for seven different locations represented by the vertical lines. a Wind-tunnel comparisons in streamwise direction along the centre of the domain (through the middle of the hill). b Corresponding streamwise velocity variances. Velocity and variance are normalized by $u_{H}$, which is the wind speed at "hill height" for a flow field without a hill $\left(4.9 \mathrm{~m} \mathrm{~s}^{-1}\right.$ for our LES)

distances from the wall. This is further confirmed when we compare streamwise velocity measurements and simulation results taken along the y-axis in the wake of the hill at two separate elevations: at $H / 8$ (Fig. 2a) and at $H$ (Fig. 2b). Due to the periodic boundary conditions, the flow develops spanwise heterogeneities, as observed by Calaf et al. (2010) and Cal et al. (2010). To eliminate these, we perform a symmetric averaging along the central line: $u_{i}(x, y, z)=u_{i}(x,-y, z)=0.5\left(u_{i}(x, y, z)+u_{i}(x,-y, z)\right)$, where the flow is along the $x$-axis and $y=0$ is the centre of the domain. The assumption of very high Reynolds number in our model can explain some mismatches seen in the model-data comparison as the Reynolds number for the wind tunnel is around 14,500. The mismatch near the ground could be either due to the wall model, or to an incorrect prediction of the separation point location. The viscous sublayer that ultimately gives rise to this separation point is not resolved in the LES, thus the separation point is difficult to predict on very smooth surfaces such as the present example. Improvements in agreement at the ground-air interface for both stress and velocity fields may be achieved by applying the smoothing technique proposed by Fang et al. (2011). The reduced accuracy on the lee side of the hill is also seen in Fig. 1b in which 

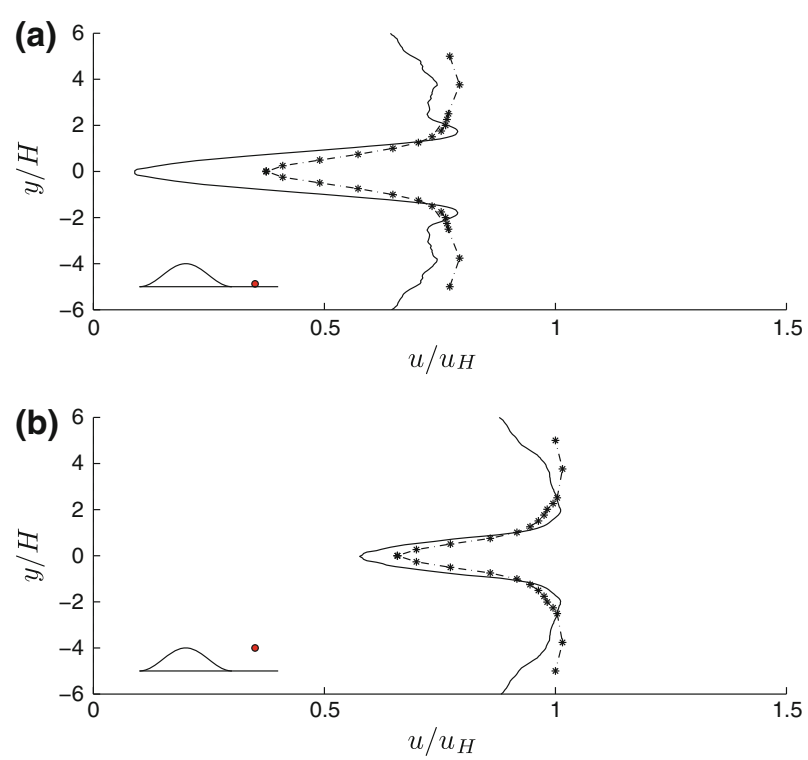

Fig. 2 Stars are the wind-tunnel measurements, the solid line represents the LES results. a Wind-tunnel comparisons of the $u$-component of velocity at $H / 8$ in the wake region at a distance $x / H=3.75$ behind the centre of the hill. $\mathbf{b}$ Wind-tunnel comparison of the $u$-component of velocity at hill height in the wake region at a distance $x / H=3.75$ behind the top of the hill. Velocity is normalized by $u_{H}$, which is the wind speed at "hill height" for a flow field without a hill $\left(4.9 \mathrm{~m} \mathrm{~s}^{-1}\right.$ for our LES)

we compare the streamwise velocity variance in the $x-z$ plane defined by $\sigma_{u}=\sqrt{\bar{u}^{\prime 2}}$. All the data are normalized by the mean velocity $u_{H}$ obtained at the hill height, $H$, in the undisturbed boundary layer. Here, the largest disagreement between the wind-tunnel measurements and LES simulation occurs in the profiles immediately downstream of the peak. This effect is expected to diminish in real topographical situations, due to the presence of sharp corners and discontinuities that enable the position of separation points to be established. This conjecture is addressed in the next section.

The impact of elevation on the accuracy of the results can be seen in Fig. 3, in which the ratios of simulated variances to the measured variances are displayed. Near the ground and up to an elevation equal to the height of the hill $(z / H \leq 1)$, and at locations upwind of the topography, the LES underpredicts the variance. Conversely the LES overpredicts the variance on the lee side of the hill $(x / H=3.75$ and $x / H=5)$, particularly for elevations between $z / H=0.5$ and $z / H=1$. All data converge at elevations above the height of the hill $(z / H \geq 1)$ where the simulated variances underestimate the measurements.

\section{The Bolund Comparison}

\subsection{The Bolund Field Campaign}

The Askervein Hill field campaign (Taylor and Teunissen 1987) was, until recently, the standard case used to test numerical simulations of turbulent flows over topography (Lopes et al. 2007; Chow and Street 2009). The Bolund field campaign, which took place from December 2008 to February 2009 on the Bolund Hill in Denmark, near the Ris $\emptyset$ Campus 


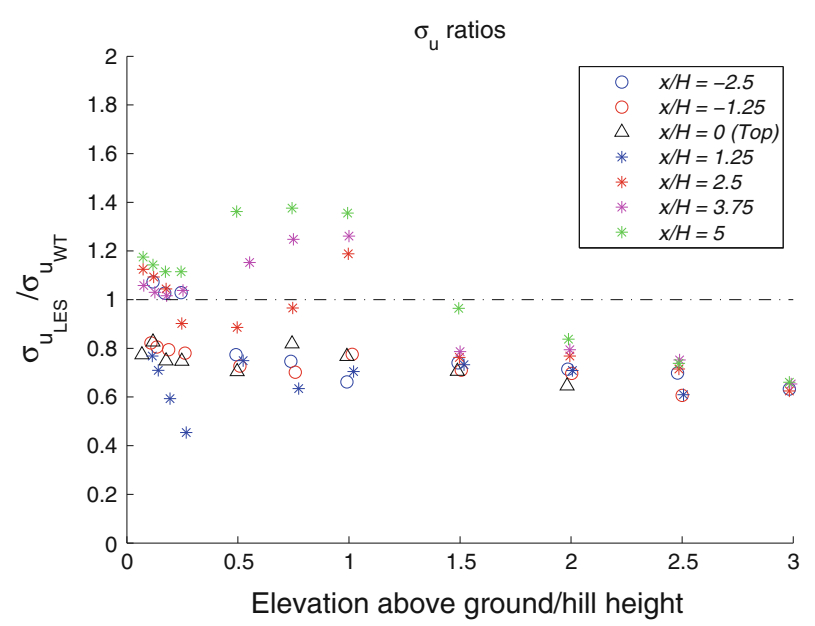

Fig. 3 Ratio of LES results to wind-tunnel data for variances of the $u$-component of velocity, results sorted by locations

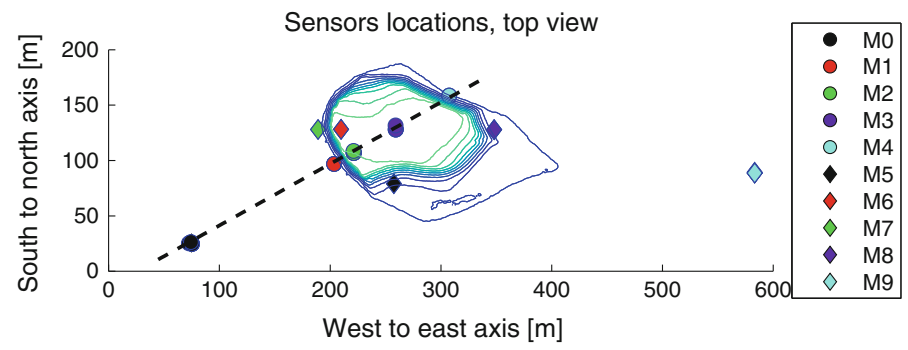

Fig. 4 Station locations on the Bolund Hill. North is upwards, the dashed line is along the $242^{\circ}$ wind direction (west to east)

of the Technical University of Denmark, has more challenging topography, including steep slopes and cliffs, than that of the Askervein Hill campaign. The details of the experiment are described in Bechmann et al. (2009). The hill is $130 \mathrm{~m}$ long (east-west axis) and $75 \mathrm{~m}$ wide (north-south axis), with a maximum height of $11.7 \mathrm{~m}$. The dominant winds are from the west and south-west. Thus the wind has an extensive upwind fetch over the sea before encountering land, leading to a "steady" flow on the windward side of the hill. The wind field first encounters a 10-m vertical cliff, after which air flows back down to sea level on the east side of the hill. A recirculation zone and a flow separation are expected due to this abrupt change of slope. During the campaign, 35 anemometers were deployed over the hill. The location of the measurement devices can be seen on Fig. 4. Instrumentation includes 23 sonic anemometers, 12 cup anemometers and two lidars. At each measurement location, the three components of the wind velocity vector and their variances were recorded for four different dominant wind directions, three westerly winds originating from the sea $\left(268^{\circ}, 254^{\circ}\right.$ and $\left.242^{\circ}\right)$ and one easterly wind originating from the land $\left(95^{\circ}\right)$. The mean wind speed during the measurements was around $10 \mathrm{~m} \mathrm{~s}^{-1}$, leading to a Reynolds number of $\operatorname{Re}=U h / v \approx 10^{7}$ (Berg et al. 2011). The measured values are 10-min averages of measurements sampled at $20 \mathrm{~Hz}$ for sonic anemometers. We followed Bechmann et al. (2009) and considered the atmosphere as neutral. 


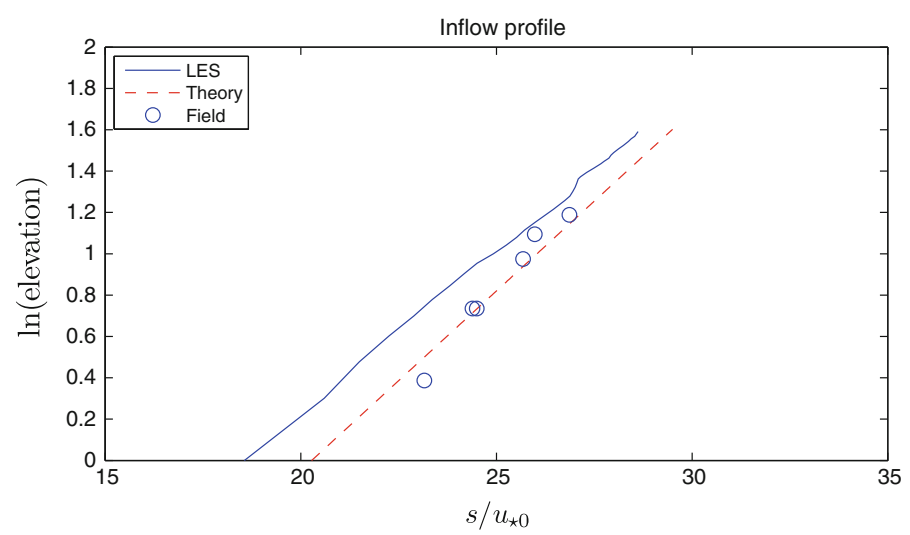

Fig. 5 Comparison of inflow wind profiles taken at mast M0

\subsection{Simulation Set-Up}

The simulations used to compute the wind fields on the Bolund Hill were performed using a domain of $512 \times 256 \times 128$ elements with dimensions of $512 \mathrm{~m} \times 256 \mathrm{~m} \times 128 \mathrm{~m}$ (wind direction, cross-wind direction and vertical direction) giving a resolution of $\mathrm{d} x=\mathrm{d} y=\mathrm{d} z=$ $1 \mathrm{~m}$. A roughness length of $z_{0}=0.3 \mathrm{~mm}$ was prescribed for the entire domain. This value is consistent with the measured surface roughness of water, but smaller than the measured roughness length over the land, viz. $z_{0}=15 \mathrm{~mm}$. It is assumed that the topography has a much larger impact on the near-surface flow dynamics than the small change in surface roughness length. Simulations were performed for the four different wind directions observed during the field campaign (i.e. wind direction from $268^{\circ}, 254^{\circ}, 242^{\circ}$ and $95^{\circ}$ ). Each simulation was run for $40,0005 \mathrm{~ms}$ timesteps, i.e. $200 \mathrm{~s}$, using a precursor simulation coupled with the buffer technique as described in Sect. 2. These settings yielded a mean running time of four advection times along the whole domain or 15 advection times along the hill.

\subsection{Comparison}

Bechmann et al. (2011) compared results from several different modelling techniques with the field measurements of the wind around the hill, but few LES results were included in these.

We first compare our results with the experiment using the same methodology used by Bechmann et al. (2011) using the data for flow along the $242^{\circ}$ axis (Fig. 4). First, the inflow wind-speed profile given by the simulations is compared with the measurements at mast M0, which is about $150 \mathrm{~m}$ upstream of the hill. The comparison can be seen in Fig. 5. The red line is the theoretical line obtained using a logarithmic profile:

$$
u\left(z_{\mathrm{agl}}\right)=\frac{u_{* 0}}{\kappa} \ln \left(\frac{z_{\mathrm{agl}}}{z_{0}}\right),
$$

where $z_{0}=0.3 \mathrm{~mm}$ is the roughness length, $\kappa=0.4$ is the von Karman constant, $u_{* 0}=0.45 \mathrm{~m} \mathrm{~s}^{-1}$ is the friction velocity measured during the field campaign and $z_{\mathrm{agl}}$ is the elevation above ground level. The points represent the field data that were used to calibrate the theoretical profile and the blue line shows the LES profile. Note that these data 

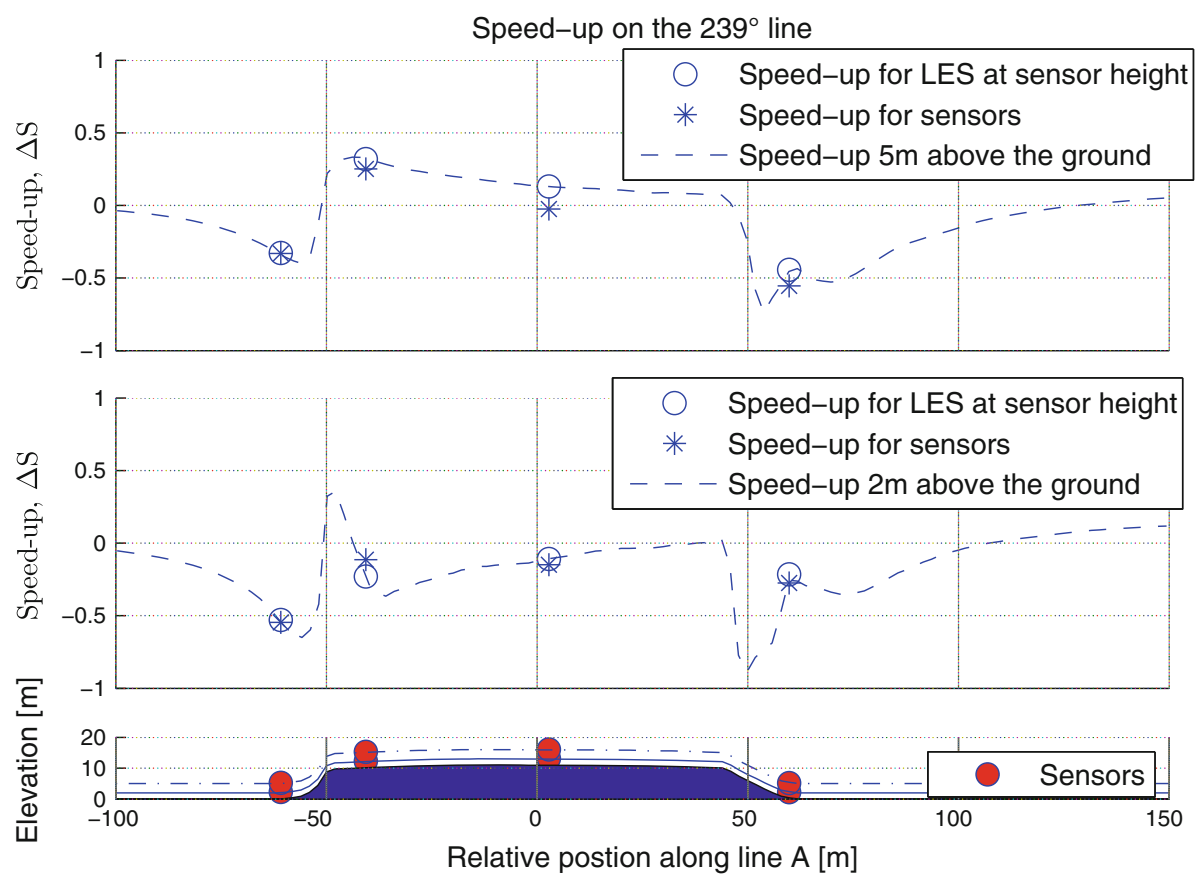

Fig. 6 Speed-up of the flow along the Bolund Hill. Wind direction is from $242^{\circ}$

are normalized by $u_{*}$. The matching is not perfect but would be considered accurate by Bechmann et al. (2011) (less than $10 \%$ error).

In order to investigate dscrepancies between simulations and measurements occuring after the first mast (M0), we follow Bechmann et al. (2011) and quantify changes in the wind field as either changes in speed (speed-up) or in direction (turning). Speed-up is defined by:

$$
\Delta S_{\mathrm{m}}=\frac{\left\langle\bar{s} / u_{* 0}\right\rangle_{z_{\mathrm{ag} l}}-\left\langle\overline{s_{0}} / u_{* 0}\right\rangle_{z_{\mathrm{ag} l}}}{\left\langle\overline{s_{0}} / u_{* 0}\right\rangle_{z_{\mathrm{agl}}}},
$$

where $\bar{s}$ is the mean wind speed at the sensor location and $\overline{s_{0}}$ is the mean wind speed at the inflow mast M0. Turning is defined as the difference between the wind direction at the measurement point and that at M0. The comparison is made for two different elevations, 2 and $5 \mathrm{~m}$ above the ground level. The results for the speed-up (Fig. 6) and for the turning of the wind (Fig. 7) both show excellent agreement with the experimental data.

\subsection{Comparison, Part II}

Excellent agreement between the EPFL-LES and the field measurements was found when using the comparison protocol of Bechmann et al. (2011), but these tests do not include all data for all wind directions. Further tests were conducted on the entire measurement set. We present here the results for the inflow velocity along the $242^{\circ}$ axis, but the other directions show similar results and are summarized in Table 1. Figure 8 shows the comparison between the LES results and all experimental data for the case of the westerly wind $\left(242^{\circ}\right)$. In this plot, the wind speeds are normalized by $u_{* 0}$, which is the friction velocity at a reference point, M0, located on the station far upstream of the hill. There is good agreement between 

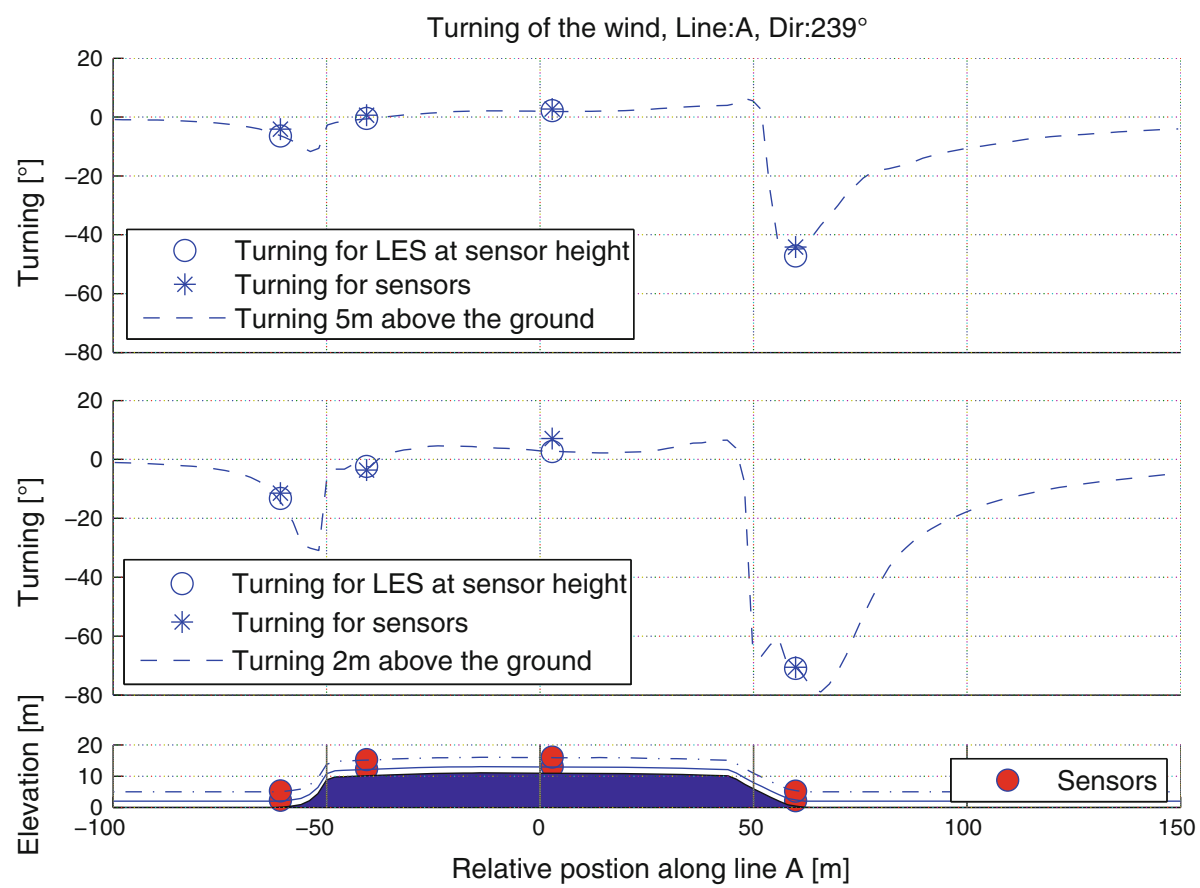

Fig. 7 Turning of the wind direction along the Bolund Hill. Wind direction is from $242^{\circ}$

Table 1 Dependence of the speed-up error (defined by Eq. 8) to the wind direction

Values in percents $(\%)$

\begin{tabular}{lccc}
\hline Wind direction & Error at $2 \mathrm{~m}$ & Error at $5 \mathrm{~m}$ & Average error \\
\hline 95 & 32.0 & 16.0 & 24.0 \\
242 & 5.7 & 8.5 & 7.1 \\
254 & 6.2 & 5.7 & 5.9 \\
268 & 9.0 & 15.2 & 12.1 \\
\hline
\end{tabular}

the field data and the LES results. In Fig. 9 the ratio of LES wind speeds to measured wind speeds is displayed as a function of elevation (Fig. 9a) and measurement location (Fig. 9b). It is clear that the largest mismatch between the LES results and the experimental data occurs at the lowest points. Many factors contribute to this lower accuracy near the surface.

1. Interpolation of the velocity field near the land surface, which is highly dependent on the wall model used;

2. The smoothing of the stress field induced by the IBM;

3. The SGS scales are more important near the surface, leading to a higher ratio of the parametrized to the resolved terms in that area.

Two of the least accurate results originate from mast M8, which is on the lee side of the hill for the $242^{\circ}$ wind direction and located on a slope parallel to the main wind direction. Mast M5 is also on a slope parallel to the $242^{\circ}$ wind direction, and mast M6, where we also see a single point of disparity, is at the top of a cliff perpendicular to the main flow. Figure 10 shows the simulated wind directions versus the measured wind directions. The point sizes 


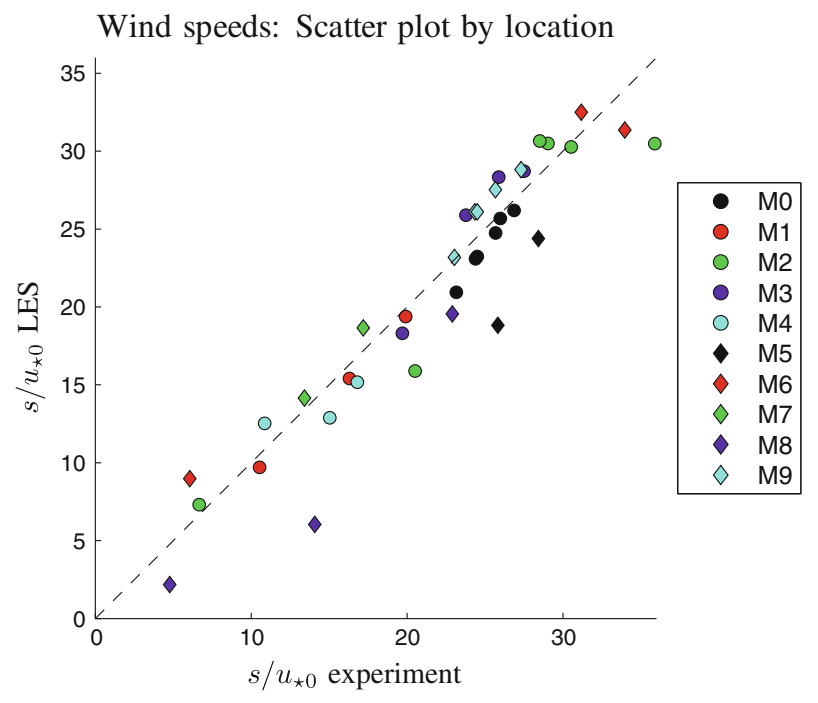

Fig. 8 Scatter plot of total speeds, field data against LES results. Wind direction is from $242^{\circ}$
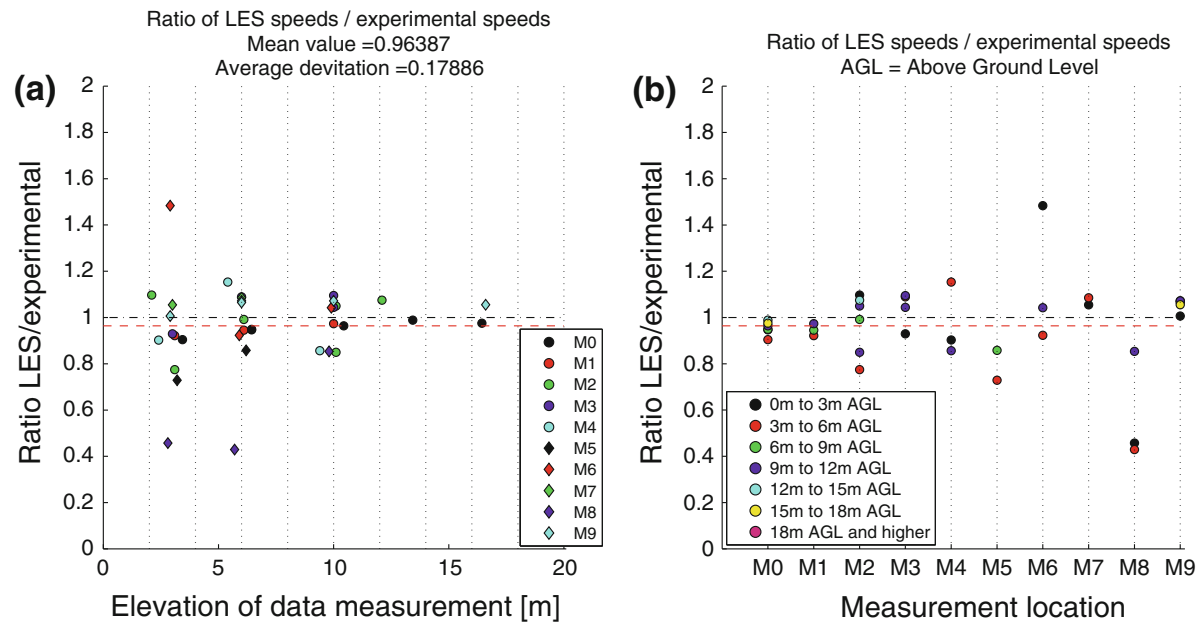

Fig. 9 Ratio of LES results to field data for total speeds, the dot colours are sorted by location (a) and elevation (b). Red line is the average value, black line is at ratio $=1$. Wind direction is from $242^{\circ}$

are proportional to the magnitude of the measured wind speed at that location. The wind speed at the mispredicted point from M8 is very low, and is once again associated with the lowest-altitude wind measurement from mast M8. The field measurements show that the wind direction at this point is parallel to the prevailing wind, but the simulation predicts it to have a wind direction rotated with respect the main flow. The results obtained for the two other sensor locations on mast M8 fit the field data with respect to the wind direction. The simulated wind direction for the middle sensor at M8 also agrees with field data (the highest sensor is a cup anemometer that only gives wind speed). Further evidence that the wind components are more difficult to capture in certain locations can be seen in Fig. 11, which 
Fig. 10 Scatter plot for wind directions, field data against LES results. The size of the dot is proportional to the total wind speed at the sensor's location. Wind direction is from $242^{\circ}$
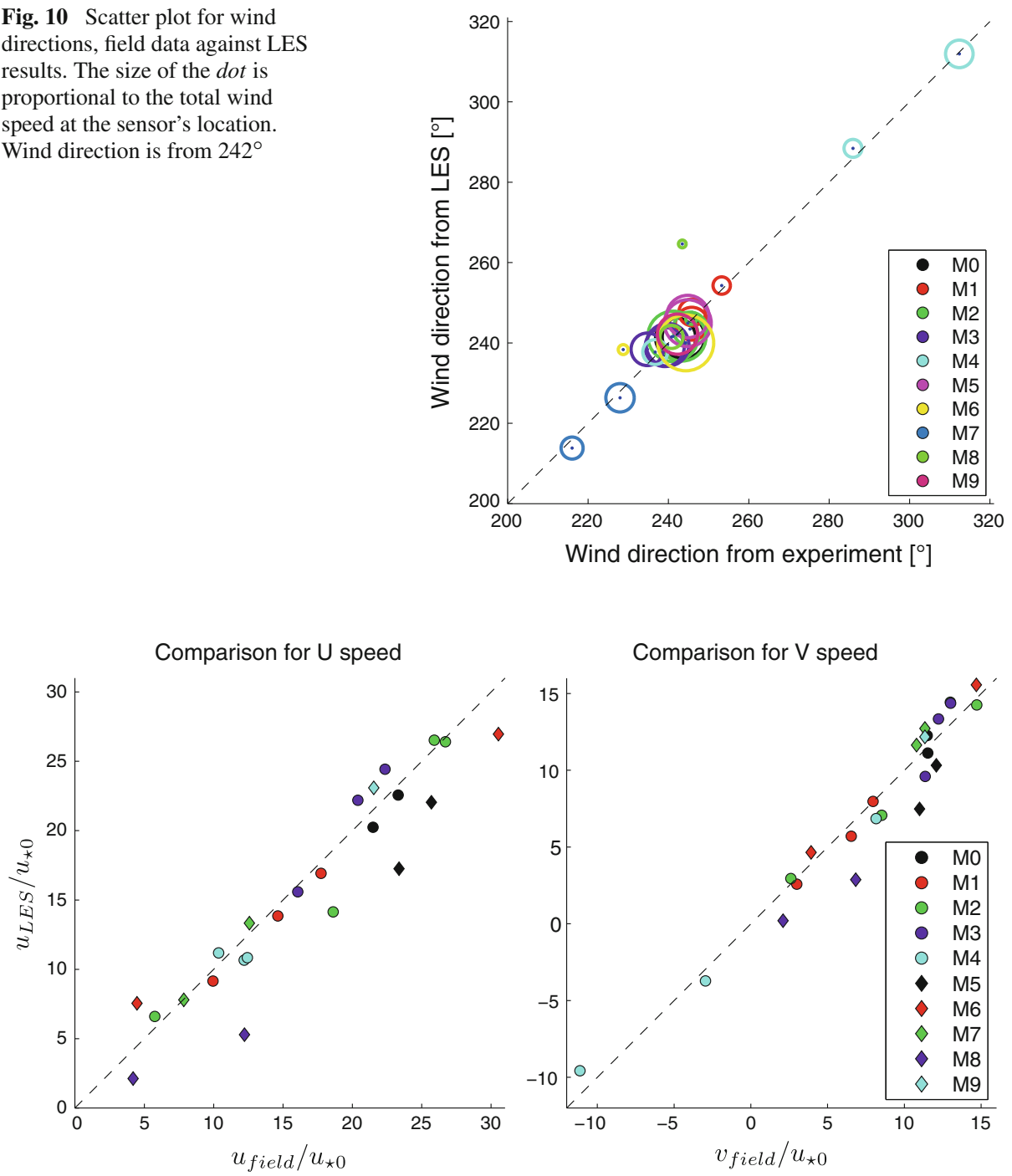

Fig. 11 Scatter plots of $u$ - and $v$-components of velocity, field data against LES results. Wind direction is from $242^{\circ}$

shows the simulated versus the measured speeds for the $u$ and $v$ components of the wind field sorted by station location. The larger discrepancy at mast M8 is once again apparent. The ratio of the simulated $u$-component to the measured $u$-component is shown in Fig. 12 . In this plot, the $v$-component is not displayed because some of the measured values are very close to zero. Greater disparity between simulations and measurements can again be seen for masts M6 and M8. Figure 13 compares the variances, $\overline{u^{\prime} u^{\prime}}$ and $\overline{v^{\prime} v^{\prime}}$, and shows the ratio of simulated to experimental data. Here, the locations with the least accurate results are situated on masts M6 and M8 for the $u$-variances and on masts M2 and M6 for the $v$-variances. At their location, on the top of the cliff, there is significant turbulence caused by orographic lifting. Note that the LES overestimates the variance for the lee side of the hill (M8) and 
Fig. 12 Ratio of LES results to field data for $u$-component of velocity, sorted by locations. Red line is the average value, black line is at ratio $=1$. Wind direction is from $242^{\circ}$

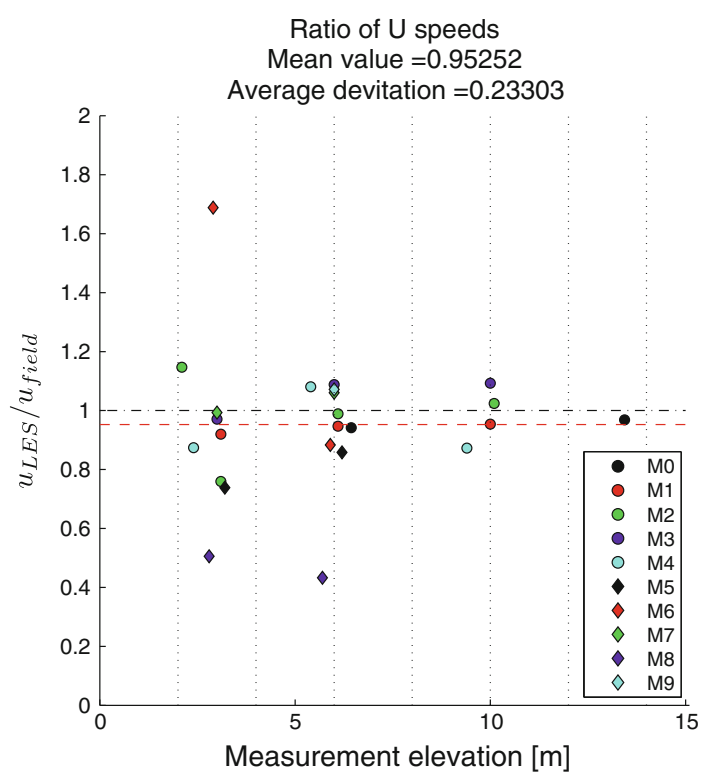

underestimates it for the other masts at the top of the cliff (M2 and M6). This trend is similar to the one observed for the wind-tunnel comparison (Fig. 3). The LES overestimates the variances on the lee side and underestimates them on the windward side and at elevations higher than the hill height.

The same analysis was conducted for the other wind directions. For winds from the sea $\left(254^{\circ}\right.$ and $\left.268^{\circ}\right)$, the results are very similar. Comparisons of the results with other wind directions verify the finding that LES is most challenged when the wind is oriented parallel to the slope. For example, for the $268^{\circ}$ wind direction, the towers M4 and M5 have lower agreement, while the mast M8 gains accuracy near the ground. Table 1 shows a numerical comparison of the results using the speed-up error, defined by:

$$
R_{\mathrm{S}}=100\left(\Delta S_{\mathrm{S}}-\Delta S_{\mathrm{m}}\right)
$$

where subscripts s and $\mathrm{m}$ denote the simulated and measured speed-up (defined by Eq. 7), respectively. With a mean error of $7.1 \%$, the $242^{\circ}$ case provides an accurate prediction of the speed-up error. The $254^{\circ}$ simulation gives the best result (mean error of $5.9 \%$ ) and the $268^{\circ}$ direction has a mean speed-up error of $12.1 \%$.

The last case, with the wind originating from the land $\left(95^{\circ}\right)$, has the largest mean speedup error $(24 \%)$. For this case, a separate inflow file was built to match the high reference shear stress $\left(u_{* 0}=0.51 \mathrm{~m} \mathrm{~s}^{-1}\right)$ and the higher surface roughness $\left(z_{0}=15 \mathrm{~mm}\right.$ compared to $z_{0}=0.3 \mathrm{~mm}$ for the cases with flow from the sea). As a result, the mean wind speed is about 1.6 times lower at heights ranging from 2 to $20 \mathrm{~m}$ above the ground than in the three first cases. For the $95^{\circ}$ case, we see the same trend as in the previous results: masts M1 and M7 located on the lee side of the hill, near the edge of the cliff, show the only inaccurate wind direction results. This mismatch is also observed when comparing wind speeds: all towers show good agreement except for the lower sensors of masts M1 and M7.

One possible source of error is the prescribed surface roughness used for the inflow file and in simulation. For example when the wind direction was $95^{\circ}$ (from land), the whole simulation was run with a single surface roughness of $z_{0}=0.3 \mathrm{~mm}$. During the blind test with flow 

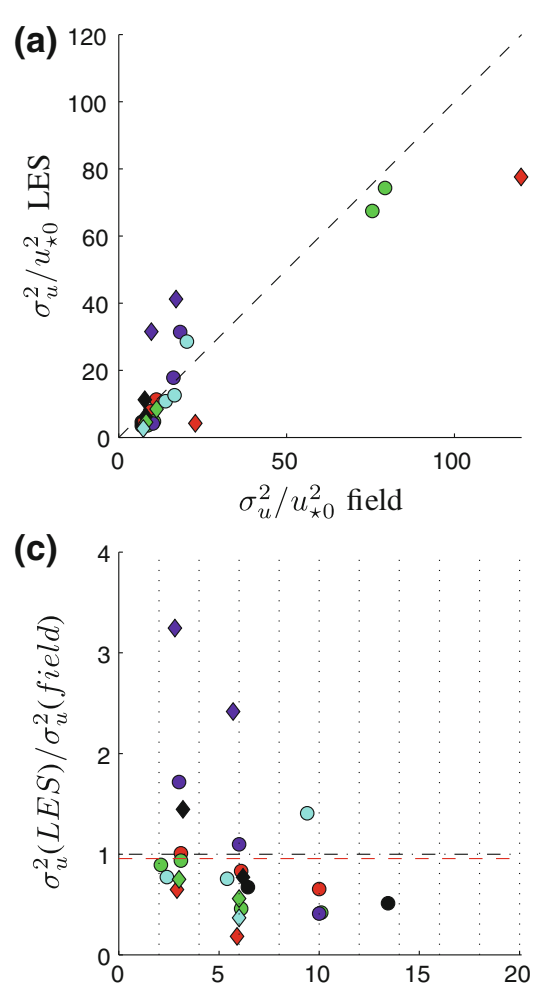

Elevation of data measurement $[\mathrm{m}]$
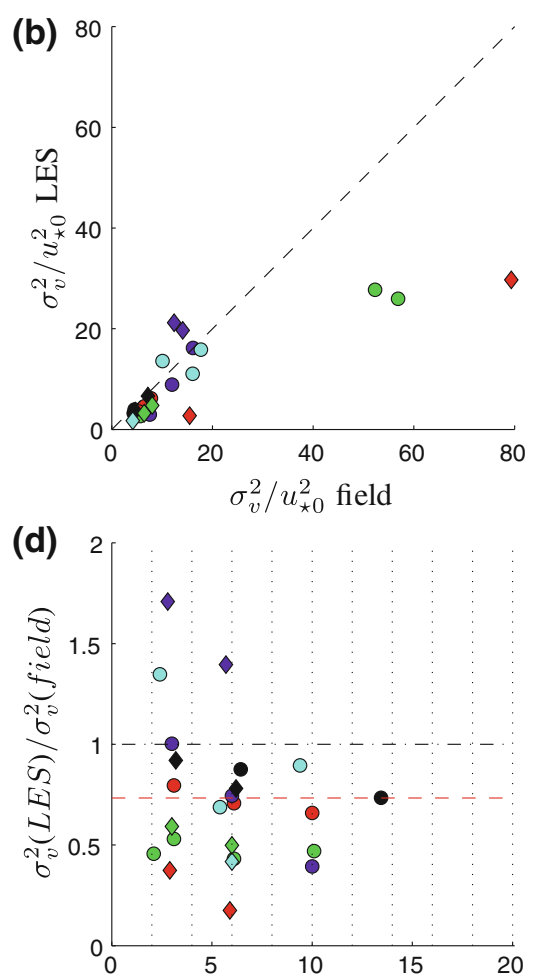

Elevation of data measurement $[\mathrm{m}]$

\begin{tabular}{lllllllllllllllllllll} 
& $\mathrm{M} 0$ & $\bigcirc$ & $\mathrm{M} 1$ & $\bigcirc$ & $\mathrm{M} 2$ & $\diamond$ & $\mathrm{M} 3$ & $\bigcirc$ & $\mathrm{M} 4$ & $\diamond$ & $\mathrm{M} 5$ & $\diamond$ & $\mathrm{M} 6$ & $\diamond$ & $\mathrm{M} 7$ & $\diamond$ & $\mathrm{M} 8$ & $\diamond$ & $\mathrm{M} 9$ \\
\hline
\end{tabular}

Fig. 13 Top Scatter plots of variances of $u$ - and $v$-components of velocity, field data against LES results. Bottom ratio of variances of $u$ - and $v$-components of velocity, LES results over field data. Red line is the average value, black line is at ratio $=1$. Wind direction is from $242^{\circ}$

from the land (inflow file with $z_{0}=15 \mathrm{~mm}$ ), the airflow also crosses water (simulation with $z_{0}=0.3 \mathrm{~mm}$ ) before interacting with the hill. Notice that the amount of water between the coast and the hill was not specified in the blind test and that a band of land emerges from the sea at low tide. The speed-up error at a height of $2 \mathrm{~m}$ has a value of $32 \%$, whereas at $5 \mathrm{~m}$ it decreases to $16 \%$. This does not follow the trend observed for flow from the sea and suggests that the boundary conditions on the ground may need to be modified. To further investigate the impact of surface roughness on the accuracy of the LES results, further simulations were performed.

\subsection{Sensitivity and Performance}

The assumption that surface roughness has a much lower importance than topography was tested by running a new simulation for the wind direction from $242^{\circ}$ using two different surface roughnesses: $z_{0}=15 \mathrm{~mm}$ for land and $z_{0}=0.3 \mathrm{~mm}$ for sea and inflow file. The sensitivity of the model to the grid resolution was also tested by running another simulation involving two different surface roughnesses but with a grid of $256 \times 128 \times 64$ cubes, i.e with half the elements in each direction, leading to a grid size of $2 \mathrm{~m} \times 2 \mathrm{~m} \times 2 \mathrm{~m}$. Table 2 
Table 2 Improvements of speed-up error (defined by Eq. 8) obtained by increasing the resolution of the grid and/or using different surfaces roughnesses for land and sea

\begin{tabular}{llclc}
\hline Size of the grid & Different $z_{0} ?$ & $\begin{array}{l}\text { Error } \\
\text { at } 2 \mathrm{~m}\end{array}$ & $\begin{array}{l}\text { Error } \\
\text { at } 5 \mathrm{~m}\end{array}$ & $\begin{array}{l}\text { Average } \\
\text { error }\end{array}$ \\
\hline $256 \times 128 \times 64$ & Yes & 14.5 & 9.2 & 11.9 \\
$512 \times 256 \times 128$ & No & 5.7 & 8.5 & 7.1 \\
$512 \times 256 \times 128$ & Yes & 4.7 & 5.6 & 5.1 \\
\hline
\end{tabular}

Values in percent $(\%)$

Table 3 Comparison between the speed-up errors (defined by Eq. 8) from models taking part in the blind test, measured data and results from the EPFL-LES model

Cases 1 and 3 represent the flow from the sea from directions $242^{\circ}$ and $268^{\circ}$. Values in percent $(\%)$

\begin{tabular}{lll}
\hline Model & All cases (best) & Case $1+3$ (best) \\
\hline Two-equation RANS & $13.6(10.2)$ & $15.1(11.4)$ \\
Experiment & & $14.7(13.3)$ \\
One-equation RANS & $16.3(12.2)$ & $17.2(13.8)$ \\
LES & $16.0(13.5)$ & $17.3(14.1)$ \\
Linearized & $21.0(18.5)$ & $23.7(20.6)$ \\
All models & 15.8 & 17.3 \\
EPFL-LES & 12.3 & 9.6 \\
\hline
\end{tabular}

presents these results. The use of two different surface roughnesses in the higher-resolution simulations lowers the mean speed-up error from 7.1 to $5.1 \%$. As expected, the use of a lowresolution grid leads to less accurate results, and a mean speed-up error of $11.9 \%$. Notice that, in this case, the error near the ground is larger than it is at $5 \mathrm{~m}$, which is the opposite of the case with a higher resolution.

The results obtained with the EPFL-LES are compared to the modeling efforts summarized by Bechmann et al. (2011) in Table 3. When all the cases are included, the EPFL-LES is among the most accurate models, and if the $95^{\circ}$ case is excluded, it shows better results than any other model.

\section{Conclusion}

To simulate the wind fields around topography, we have implemented an immersed boundary method within a large-eddy simulation model (LES).

Results were first tested against wind-tunnel data. For this case, we see good agreement between simulation and experimental data, except for some inaccuracies near the ground and on the lee side of the hill; these are likely due to the fact that the separation point is hard to resolve on such a smooth surface. The EPFL-LES is further tested against experimental data from the Bolund Hill field campaign. A comparison is made for four main measured wind directions, with the focus on the wind speed, direction and variance. In general, the LES shows good agreement with field measurements, however the points closest to steep slopes aligned parallel to the main wind direction or near the ground appear to be the most difficult to predict accurately. Nevertheless, the results are still favourable. The observed disparities indicate that there is room for improvement in the wall model used as boundary condition for ground, where LES predictions are difficult. A sensitivity study confirms the importance of 
using appropriate surface roughness for the sea and the land surfaces and shows that a coarse grid significantly lowers accuracy near the ground. The comparison of our results with other approaches presented in Bechmann et al. (2011) is very favourable.

Acknowledgments We would like to thank the collaborators who participated in the Bolund Experiment and provided us with the data for comparison. We are also grateful to the Swiss National Supercomputing Centre (CSCS) for the use of their computers. We would like to thank the Swiss National Science Foundation for their financial support under grant 200021-120238. Mary Parlange provided great assistance with english ameliorations. Finally, we thank the reviewers for their valuable suggestions in improving this article.

\section{References}

Albertson JD, Parlange MB (1999) Surface length scales and shear stress: implications for land-atmosphere interaction over complex terrain. Water Resour Res 35(7):2121-2132

Allen T, Brown AR (2002) Large-eddy simulation of turbulent separated flow over rough hills. Boundary-Layer Meteorol 102(2):177-198

Andren A, Brown AR, Graf J, Mason PJ, Moeng CH, Nieuwstadt FTM, Schumann U (1994) Large-eddy simulation of a neutrally stratified boundary-layer-a comparison of 4 computer codes. Q J R Meteorol Soc 120(520):1457-1484

Balaras E (2004) Modeling complex boundaries using an external force field on fixed Cartesian grids in large-eddy simulations. Comput Fluids 33(3):375-404

Bechmann A, Berg J, Courtney MS, Jørgensen HE, Mann J, Sørensen NN (2009) The Bolund experiment: overview and background. Technical report, Riso DTU, National Laboratory for Sustainable Energy, Denmark

Bechmann A, Sorensen NN, Berg J, Mann J, Rethore PE (2011) The Bolund experiment, Part II: blind comparison of microscale flow models RID C-6255-2011 RID C-5528-2011. Boundary-Layer Meteorol 141(2):245-271

Berg J, Mann J, Bechmann A, Courtney MS, Jorgensen HE (2011) The Bolund experiment, Part I: flow over a steep, three-dimensional Hill RID C-6255-2011. Boundary-Layer Meteorol 141(2):219-243

Bou-Zeid E, Meneveau C, Parlange MB (2004) Large-eddy simulation of neutral atmospheric boundary layer flow over heterogeneous surfaces: blending height and effective surface roughness RID A-9796-2008 RID A-3319-2010. Water Resour Res 40(2):W02505

Bou-Zeid E, Meneveau C, Parlange M (2005) A scale-dependent Lagrangian dynamic model for large eddy simulation of complex turbulent flows RID A-9796-2008 RID A-3319-2010. Phys Fluids 17(2):025105

Brown AR, Hobson JM, Wood N (2001) Large-eddy simulation of neutral turbulent flow over rough sinusoidal ridges. Boundary-Layer Meteorol 98(3):411-441

Cal RB, Lebron J, Castillo L, Kang HS, Meneveau C (2010) Experimental study of the horizontally averaged flow structure in a model wind-turbine array boundary layer. J Renew Sust Energy 2(1):013106

Calaf M, Meneveau C, Meyers J (2010) Large eddy simulation study of fully developed wind-turbine array boundary layers. Phys Fluids 22(1):015110

Chester S, Meneveau C, Parlange MB (2007) Modeling turbulent flow over fractal trees with renormalized numerical simulation. J Comput Phys 225(1):427-448

Chow FK, Street RL (2009) Evaluation of turbulence closure models for large-eddy simulation over complex terrain: flow over Askervein Hill. J Appl Meteorol Clim 48(5):1050-1065

Cristallo A, Verzicco R (2006) Combined immersed boundary/large-eddy-simulations of incompressible three dimensional complex flows. Flow Turbul Combust 77(1-4):3-26

Deardorff JW (1970) A numerical study of 3 dimensional turbulent channel flow at large Reynolds numbers. J Fluid Mech 41:453

Fang JN, Diebold M, Higgins C, Parlange MB (2011) Towards oscillation-free implementation of the immersed boundary method with spectral-like methods. J Comput Phys 230(22):8179-8191

Ferziger J, Péric M (1999) Computational methods for fluid dynamics. Springer, Berlin 426 pp

Gal-Chen T, Somerville RCJ (1975) Numerical-solution of Navier-Stokes equations with topography. J Comput Phys 17(3):276-310

Germano M, Piomelli U, Moin P, Cabot WH (1991) A dynamic subgrid-scale eddy viscosity model. Phys Fluids A 3(7):1760-1765

Ishihara T, Hibi K, Oikawa S (1999) A wind tunnel study of turbulent flow over a three-dimensional steep hill. J Wind Eng Ind Aerodyn 83:95-107 
Lilly DK (1967) The representation of small-scale turbulence in numerical simulation experiments. In: The IBM scientific computing symposium on environmental sciences

Lopes AS, Palma JMLM, Castro FA (2007) Simulation of the askervein flow. Part 2: large-eddy simulations. Boundary-Layer Meteorol 125(1):85-108

Loureiro JBR, Monteiro AS, Pinho FT, Freire AP (2009) The effect of roughness on separating flow over two-dimensional hills. Exp Fluids 46(4):577-596

Meneveau C, Katz J (2000) Scale-invariance and turbulence models for large-eddy simulation. Annu Rev Fluid Mech 32:1-32

Mittal R, Iaccarino G (2005) Immersed boundary methods. Annu Rev Fluid Mech 37:239-261

Nieuwstadt F, Mason P, Moeng C, Schumann U (1991) Large-eddy simulation of the convective boundary layer: a comparison of four computer codes. In: 8th symposium on turbulent shear flows

Peskin CS (1972) Flow patterns around heart valves-numerical method. J Comput Phys 10(2):252-271

Peskin CS (2002) The immersed boundary method. Acta Numer 11:1-39

Porte-Agel F, Meneveau C, Parlange MB (2000) A scale-dependent dynamic model for large-eddy simulation: application to a neutral atmospheric boundary layer. J Fluid Mech 415:261-284

Saad Y (1981) Krylov subspace methods for solving large unsymmetric linear-systems. Math Comput 37(155):105-126

Smagorinsky J (1963) General circulation experiments with the primitive equations: I. The basic experiment. Mon Weather Rev 91:99-164

Tamura T, Cao S, Okuno A (2007) LES study of turbulent boundary layer over a smooth and a rough 2D hill model. Flow Turbul Combust 79(4):405-432

Taylor PA, Teunissen HW (1987) The Askervein Hill project-overview and background data. BoundaryLayer Meteorol 39(1-2):15-39

Tseng YH, Ferziger JH (2003) A ghost-cell immersed boundary method for flow in complex geometry. J Comput Phys 192(2):593-623

Tseng YH, Meneveau C, Parlange MB (2006) Modeling flow around bluff bodies and predicting urban dispersion using large eddy simulation. Environ Sci Technol 40(8):2653-2662

Voller VR, Porte-Agel F (2002) Moore's law and numerical modeling. J Comput Phys 179(2):698-703

Yue WS, Parlange MB, Meneveau C, Zhu WH, van Hout R, Katz J (2007) Large-eddy simulation of plant canopy flows using plant-scale representation. Boundary-Layer Meteorol 124(2):183-203 